\title{
Presenteeism and Associated Factors Among Nursing Personnel with Low Back Pain: A Cross-Sectional Study
}

This article was published in the following Dove Press journal: Journal of Pain Research

\author{
Takahiko Yoshimoto $\mathbb{D}^{1,2}$ \\ Hiroyuki Oka (DD ${ }^{2}$ \\ Hirotaka Ochiai (D) \\ Shuhei Ishikawa ${ }^{3}$ \\ Akatsuki Kokaze (D) \\ Shingo Muranaga ${(\mathbb{D})^{3}}^{3}$ \\ Ko Matsudaira $\mathbb{D}^{2}$
}

'Department of Hygiene, Public Health and Preventive Medicine, Showa University School of Medicine, Shinagawa-ku, Tokyo, Japan; ${ }^{2}$ Department of Medical Research and Management for Musculoskeletal Pain, 22nd Century Medical \& Research Center, Faculty of Medicine, The University of Tokyo, Bunkyo-ku, Tokyo, Japan; ${ }^{3}$ Department of Rehabilitation, Kameda Medical Center, Kamogawa, Chiba, Japan
Correspondence: Takahiko Yoshimoto Department of Hygiene, Public Health and Preventive Medicine, Showa University School of Medicine, I-5-8 Hatanodai, Shinagawa-ku, Tokyo 142-8555, Japan

Tel +8I (3)3784-8I34

Fax +8I (3)3784-7733

Email yoshimotot@med.showa-u.ac.jp
Background: A decrease in work productivity due to presenteeism among healthcare workers with low back pain (LBP) is a major problem in the workplace. It is important to determine the factors associated with presenteeism to successfully manage work productivity among nursing staff with LBP. This study aimed to identify the factors associated with presenteeism among nursing personnel with LBP through the evaluation of several aspects, including individual, occupational, and psychological factors.

Methods: We conducted a cross-sectional study with 668 nursing personnel who had experienced LBP within the 4 weeks before study enrollment at a tertiary hospital in Japan. Information on demographics (eg, sex, age, height, weight, etc.), LBP intensity (Numerical Rating Scale, NRS), kinesiophobia (Tampa Scale for Kinesiophobia-11, TSK11), depressive condition (K6), workaholism, overworking hours, frequency of shift work, sleep problem, work-related stress, and presenteeism (Work Productivity and Activity Impairment-General Health) were collected using a self-administered questionnaire. Multiple linear regressions were applied to examine the factors related to presenteeism. We further used a multiple imputation by chained equations for missing data in the model. Results: Multiple linear regression analysis after adjusting for covariates showed that NRS (regression coefficient $\beta=2.275)$, TSK-11 (1.112), K6 (0.616), and sleep duration ( -1.990$)$ were significantly associated with presenteeism. These results with complete-case analyses were similar to those with multiple imputation analyses.

Conclusion: Psychological factors, such as kinesiophobia and depressive symptoms, were associated with presenteeism independently of LBP intensity among nursing staff with LBP. Our findings suggest that the above-mentioned factors may need to be considered for the development of strategies to increase work productivity among nursing staff with LBP.

Keywords: low back pain, presenteeism, productivity, nurse, psychological factor

\section{Introduction}

Low back pain (LBP) is an extremely common symptom worldwide and is highly prevalent in working populations. ${ }^{1}$ The 1 -year prevalence of LBP has been reported to be $38 \%$, and most people experience LBP in their life. ${ }^{2}$ LBP incurs enormous costs for treatment and loss of work productivity, which results in a negative socioeconomic impact. Among the total LBP-related costs, the costs of lost productivity are much greater than medical/pharmacy costs. ${ }^{3}$ The productivity loss of workers includes not only sick leave (referred to as absenteeism), but also reduced productivity while being at work (referred to as presenteeism). Several studies have indicated that presenteeism

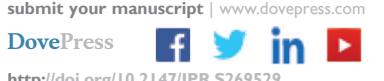

http://doi.org/10.2147/IPR.S269529
Journal of Pain Research 2020:13 2979-2986 
is a major component of the total costs related to health conditions. ${ }^{4,5}$ Moreover, it has been reported that presenteeism is a risk factor for future absenteeism. ${ }^{6}$ Therefore, it is crucial to improve the loss of work productivity due to presenteeism among individuals who suffer from LBP.

Furthermore, LBP is more prevalent among nursing staff than among other occupational staff or in the general population. ${ }^{7,8}$ Nurses with LBP often have psychological problems (eg, depression) or work-related stress, ${ }^{9,10}$ which may be related to productivity loss in the workplace. However, information on factors associated with presenteeism among nursing staff with LBP is scarce.

Several studies have indicated that an increasing severity of LBP was significantly associated with greater presenteeism. $^{11,12}$ With regard to psychological aspects, a previous study indicated that chronic LBP patients with depression experienced a greater reduction in work productivity than those without depression. ${ }^{13}$ Moreover, a recent study in eldercare workers indicated that painrelated fear was a key factor for presenteeism among workers with LBP. ${ }^{14}$ Pain-related fear including kinesiophobia has been suggested as a potential factor in the development and persistence of LBP. ${ }^{15}$

To successfully manage work productivity among nursing staff with LBP, it is essential to determine the factors associated with presenteeism from multidimensional perspectives. The aim of the present study was to identify the factors related to presenteeism among nursing personnel with LBP through an evaluation of several aspects, including individual, occupational, and psychological factors.

\section{Materials and Methods}

\section{Study Population}

We analyzed data from a cross-sectional survey among nursing personnel at the Kameda Medical Center in Chiba, Japan during February 2017. ${ }^{16}$ Occupational health staff distributed a self-administered questionnaire to all the nursing personnel $(\mathrm{n}=1,152)$. The participants responded anonymously to the questionnaire, placed the filled-out questionnaire in an envelope, and sealed it. Then, the health staff collected and forwarded the completed questionnaires to the authors. All participants provided written informed consent. This study protocol was approved by the Research Ethics Committee of Kameda Medical Center (approval no. 16-159).

LBP was defined as pain in the lower back area lasting for more than 1 day in the previous 1 month. ${ }^{17}$ Pain related to menstruation, pregnancy, or a common cold were excluded. An illustration depicting the low back area (between the lower costal margin and the gluteal folds) was included in the questionnaire. ${ }^{18}$ We included all workers who responded that they had experienced LBP during the past 1 month.

\section{Data Collection}

Each participant was required to complete the questionnaire with regard to demographic information (eg, sex, age, height, weight, etc.), LBP intensity, kinesiophobia, depressive condition, workaholism, overworking hours, frequency of shift work, sleep problem, work-related stress, and work productivity (presenteeism). The body mass index (BMI) was calculated as the weight $(\mathrm{kg})$ divided by the height $(\mathrm{m})$ squared.

\section{Independent Variables}

The intensity of LBP experienced during the 4 weeks preceding study enrollment was evaluated using a numerical rating scale (NRS; scores from 0 [no pain] to 10 [worst pain imaginable]).

Kinesiophobia was assessed using the short version of the Tampa Scale for Kinesiophobia (TSK-11). The Japanese version of the TSK-11 has been validated in previous studies. ${ }^{19,20}$ The TSK-11 comprises 11 items, and each item is rated on a 4-point Likert scale (1: strongly disagree, 4: strongly agree). The score was summed (range: 11 to 44), and higher total scores reflected a greater degree of painrelated fear of movement/(re)injury.

To evaluate the depressive condition, we used the Kessler Screening Scale for Psychological Distress, referred to as $\mathrm{K}^{2} .^{21}$ The scale comprises six items that measure the following psychological distress experienced during the past 30 days: nervousness, hopelessness, irritability, negative affect, fatigue, and worthlessness. Each item was scored on a 5-point Likert scale (0: none of the time, 4: all of the time). The Japanese version of the K6 was developed by Furukawa et al, and demonstrated acceptable reliability and validity (Cronbach's $\alpha 0.85){ }^{22}$

We evaluated workaholism by using the Dutch Workaholism Scale, ${ }^{23}$ which consists of 10 items on excessive working and obsession with work. Each item was scored on a 4-point rating scale, with scores ranging from 1 (totally disagree) to 4 (totally agree). This scale has been confirmed to have reliability and validity in the Dutch and Japanese populations. $^{23}$

The overworking duration was evaluated in a selfreported form: "how many hours did you work beyond 
the regular hours (normal working hours) during the past month?". Participants were asked to evaluate the frequency of shift work by the following question: "how many night-shifts did you work during the past month?".

Sleep problems were evaluated by a self-administered questionnaire on parameters that included sleep duration and sleep habits in the past month. ${ }^{24}$ With regard to the sleep duration, participants were asked the following question: "On average, how much did you sleep per day during the past 4 weeks?" On sleep habits, taking longer than 30 min to fall asleep was considered to indicate difficulty initiating sleep. Three times or more per week of nocturnal awakenings or early morning awakenings were defined as difficulty maintaining sleep and early morning awakening, respectively. Insomnia was defined as present if the participants reported at least one of the three abovementioned symptoms. $^{24}$

The Brief Job Stress Questionnaire (BJSQ) ${ }^{25}$ was used to evaluate work-related stress. The questionnaire comprises 57 items which were derived from several standard questionnaires, such as National Institute for Occupational Safety and Health, ${ }^{26}$ the Job Content Questionnaire, ${ }^{27}$ and the Subjective Well-being Inventory. ${ }^{28}$ Among the 19 work-related stress factors assessed in the BJSQ, we measured the following factors based on the job demand-control-support model: quantitative job demand (three items), qualitative job demand (three items), job control (three items), support from supervisors (three items), and support from coworkers (three items). For each abovementioned factor, a standardized score with a 5-point scale (1: lowest, 5: highest) was developed on the basis of data from over 10,000 workers. The highest or lowest (different by question) scores of the scale were considered to indicate stress. $^{29}$ The psychometric properties of the BJSQ have been confirmed. ${ }^{30}$

\section{Dependent Variables}

Presenteeism was assessed with the Work Productivity and Activity Impairment-General Health (WPAI-GH). ${ }^{31}$ The WPAI-GH has been assessed for construct validity and reproducibility. ${ }^{31}$ Participants were asked the following question: "During the past seven days, how much did your health problems affect your productivity while you were working?". Responses were provided on a 11-point rating scale ranging from 0 (health problems had no effect on my work) to 10 (health problems completely prevented me from working). The score was then multiplied by 10 to create a percentage of impairment, with higher numbers indicating greater impairment and less productivity.
Information on absenteeism was collected based on the following question: "How many days have you been absent from work because of LBP during the past 12 months?". Absenteeism was considered if respondents mentioned absence from work for 1 or more days.

\section{Statistical Analysis}

Data on participant characteristics are indicated as median (25th, 75th percentiles) for continuous variables or as numbers (\%) for categorical variables. Moreover, Spearman correlation coefficients between explanatory variables and presenteeism level were calculated. To assess the independent association of each factor with presenteeism, multiple linear regression analysis was employed, with the WPAI-GH score as a dependent variable. After a crude analysis, a multivariable analysis was conducted after adjusting for age, sex, BMI, job demand (quantitative, qualitative), job control, social support (supervisors, coworkers), NRS, TSK-11, K6, workaholism, overworking hours, frequency of shift work, sleep duration, and insomnia.

Furthermore, we used multiple imputation to address a potential bias in missing data under the missing at random assumption. ${ }^{32}$ A total of 20 imputed datasets were created using multiple imputation by chained equations (MICE). ${ }^{33}$ In the imputation process, all variables used in the present study, except NRS, were included. The estimates, which were calculated on multiple linear regression analysis for each imputed dataset, were then combined using Rubin's rules to obtain the integrated estimates. ${ }^{34}$ A two-sided $p$-value $<0.05$ was considered statistically significant. All statistical analyses were conducted using SAS version 9.4 (SAS Institute Inc, Cary, NC, USA).

\section{Results}

In total, 1075 (response rate: 93.3\%) nursing personnel responded to the self-administered questionnaire. Among all respondents, $62.1 \%$ had experienced LBP during the preceding 4 weeks $(n=668)$. More than $80 \%$ of the study participants were women, and the median age was 32.0 years (Table 1). The median intensity of LBP that they experienced was 3.0, as measured by the NRS. The degree of presenteeism (0-100) was 30 . Only $3.3 \%$ of subjects had been absent from work because of LBP within the past year.

Table 2 presents the correlation between presenteeism and several variables. The intensity of LBP $(p<0.001)$, TSK-11 $(p<0.001)$, K6 $(p<0.001)$, workaholism $(p<0.001)$, overworking hours $(p=0.016)$, and frequency of shift work $(p=0.015)$ were positively correlated with 
Table I Characteristics of the Participants in the Present Study $(n=668)$

\begin{tabular}{|c|c|c|}
\hline Variables & Values & $\begin{array}{l}\text { No. of } \\
\text { Missing } \\
\text { Data }\end{array}$ \\
\hline Sex (women), n (\%) & $554(83.2 \%)$ & 2 \\
\hline Age, years & $32.0(24.0,42.0)$ & 14 \\
\hline Body mass index, $\mathrm{kg} / \mathrm{m}^{2}$ & $21.2(19.5,23.6)$ & 50 \\
\hline Low back pain intensity & $3.0(2.0,4.0)$ & 0 \\
\hline Tampa Scale for Kinesiophobia-II & $22(18,25)$ & 21 \\
\hline K6 & $5(2,10)$ & 10 \\
\hline Workaholism & $21(17,25)$ & 18 \\
\hline Overworking, hours & $10.0(3.0,20.0)$ & 55 \\
\hline Frequency of shift work, per month & $5(0,10)$ & 31 \\
\hline Sleep duration, hours & $6.0(5.0,7.0)$ & 6 \\
\hline Insomnia, n (\%) & $300(45.5 \%)$ & 9 \\
\hline Quantitative job demand, n (\%) & & 3 \\
\hline Not stressed & $396(59.5 \%)$ & \\
\hline Stressed & $269(40.5 \%)$ & \\
\hline Qualitative job demand, $n$ (\%) & & 3 \\
\hline Not stressed & $223(33.5 \%)$ & \\
\hline Stressed & $442(66.5 \%)$ & \\
\hline Job control, n (\%) & & 3 \\
\hline Not stressed & $558(83.9 \%)$ & \\
\hline Stressed & $107(16.1 \%)$ & \\
\hline Support from supervisor, n (\%) & & 3 \\
\hline Supported & $580(87.2 \%)$ & \\
\hline Not supported & $85(12.8 \%)$ & \\
\hline Support from coworker, n (\%) & & 3 \\
\hline Supported & 446 (67.1\%) & \\
\hline Not supported & $219(32.9 \%)$ & \\
\hline Presenteeism & $30(10,50)$ & 6 \\
\hline Absenteeism, n (\%) & $22(3.3 \%)$ & 52 \\
\hline
\end{tabular}

Note: Values are presented as median (25th, 75 th percentile), except where indicated as $n(\%)$.

the degree of presenteeism. However, sleep duration $(p=$ 0.003 ) was negatively correlated with presenteeism.

We conducted linear regression analysis to evaluate the impact of several individual factors on presenteeism (Table 3). After adjusting for several confounders in the complete-case data, multiple linear regression analysis indicated that NRS, TSK-11, K6, and sleep duration were significantly associated with presenteeism. The influence of explanatory variables on the outcome of interest was greatest in the TSK-11 (standardized regression coefficient $=0.256)$ when compared with the NRS (0.162), K6 (0.131), and sleep duration $(-0.091)$. We undertook
Table 2 Spearman Correlation Coefficients Between Presenteeism and Other Variables

\begin{tabular}{|l|l|l|}
\hline & $\mathbf{r}$ & $\boldsymbol{p}$ value \\
\hline Low back pain intensity & 0.248 & $<0.001$ \\
Tampa Scale for Kinesiophobia-II & 0.296 & $<0.001$ \\
K6 & 0.269 & $<0.001$ \\
Workaholism & 0.232 & $<0.001$ \\
Overworking hours & 0.105 & 0.016 \\
Frequency of shift work & 0.106 & 0.015 \\
Sleep duration & -0.131 & 0.003 \\
\hline
\end{tabular}

Note: Data are based on complete-case analysis $(n=527)$.

a multiple regression analysis with MICE as a sensitivity analysis, and the results were similar to those in the complete-case analysis (Table 3 ).

\section{Discussion}

The present study investigated the associations between presenteeism and several factors, including individual, work-related, and psychological aspects, among nursing personnel with LBP at a medical center. In the multiple regression analysis, LBP intensity, kinesiophobia, depressive symptoms, and sleep duration were significantly associated with presenteeism. A sensitivity analysis using MICE supported the main findings. To the best of our knowledge, this is the first study to indicate an independent association of these factors with work productivity among nursing personnel with LBP.

Our results indicated that LBP intensity, assessed by the NRS, was positively associated with presenteeism. This finding suggesting that work performance declined when pain intensity increased could be easily inferred at the workplace. Previous studies in several countries have demonstrated that increasing pain severity was associated with a significantly lower level of work productivity (greater presenteeism) among individuals with LBP. ${ }^{11,12,35}$ These previously reported findings provide a reasonable credence for the results of the present study.

We found that pain-related fear, evaluated as kinesiophobia, was significantly associated with presenteeism among nursing personnel. Moreover, it was an interesting result that kinesiophobia had a greater impact on presenteeism than the other explanatory variables. A recent systematic review reported that the degree of kinesiophobia predicted the progression of disability in people with musculoskeletal pain, with moderate evidence. ${ }^{15}$ The results of the present study were similar to those of previous studies 
Table 3 Linear Regression Analysis for Presenteeism Among Nursing Staff with Low Back Pain

\begin{tabular}{|c|c|c|c|c|c|c|c|c|c|}
\hline & \multicolumn{3}{|c|}{ Crude Analysis } & \multicolumn{3}{|c|}{$\begin{array}{l}\text { Adjusted Analysis with Complete } \\
\text { Case Data* }\end{array}$} & \multicolumn{3}{|c|}{$\begin{array}{l}\text { Adjusted Analysis with Multiple } \\
\text { Imputed Data* }\end{array}$} \\
\hline & $\boldsymbol{\beta}$ & SE & $p$ & $\boldsymbol{\beta}$ & SE & $p$ & $\boldsymbol{\beta}$ & SE & $p$ \\
\hline Low back pain intensity & 3.774 & 0.590 & $<0.001$ & 2.275 & 0.575 & $<0.001$ & 2.681 & $0.5 \mathrm{II}$ & $<0.001$ \\
\hline Tampa Scale for Kinesiophobia-II & 1.389 & 0.180 & $<0.001$ & 1.112 & 0.186 & $<0.001$ & 0.962 & 0.170 & $<0.001$ \\
\hline K6 & 1.370 & 0.197 & $<0.001$ & 0.616 & 0.215 & 0.004 & 0.674 & 0.192 & $<0.001$ \\
\hline Workaholism & 1.055 & 0.179 & $<0.001$ & 0.278 & 0.211 & 0.188 & 0.292 & 0.179 & 0.104 \\
\hline Overworking hours & 0.230 & 0.100 & 0.022 & 0.065 & 0.099 & 0.510 & -0.021 & 0.093 & 0.823 \\
\hline Frequency of shift work & 0.354 & 0.171 & 0.039 & 0.210 & 0.165 & 0.206 & 0.159 & 0.148 & 0.282 \\
\hline Sleep duration & -2.45 I & 0.945 & 0.010 & -1.990 & 0.874 & 0.023 & -1.652 & 0.778 & 0.034 \\
\hline Insomnia & 6.472 & 2.055 & 0.002 & 1.682 & 1.925 & 0.383 & 0.785 & 1.716 & 0.647 \\
\hline Quantitative job demand & 7.294 & 2.094 & $<0.001$ & 0.258 & 2.199 & 0.907 & 0.457 & 1.952 & 0.815 \\
\hline Qualitative job demand & 7.421 & 2.160 & $<0.001$ & 3.806 & 2.277 & 0.095 & 4.538 & 2.047 & 0.027 \\
\hline Job control & 2.228 & 2.942 & 0.449 & -1.216 & 2.808 & 0.665 & -2.049 & 2.394 & 0.392 \\
\hline Support from supervisor & 11.246 & 3.150 & $<0.001$ & 4.518 & 3.060 & 0.140 & 5.207 & 2.698 & 0.054 \\
\hline Support from coworker & 6.483 & 2.198 & 0.003 & 3.002 & 2.070 & 0.148 & 1.780 & 1.856 & 0.338 \\
\hline
\end{tabular}

Note: *Adjusted for age, sex, body mass index, and all variables indicated in the table.

Abbreviations: $\beta$, parameter estimate (unstandardized regression coefficient); SE, standard error.

among workers who did not undertake physically demanding tasks ${ }^{12}$ or those who provide care for the elderly; ${ }^{14}$ therefore, an excessive pain-related fear of movement/(re) injury may be a significant predictor of reduced work productivity, regardless of occupation type. In the present study, there was a significant relationship between kinesiophobia and presenteeism that was observed independently of LBP intensity itself. These findings suggest the importance of assessment and intervention in kinesiophobia for improving work productivity. A recent study with kinematic analysis indicated that a specific lumbar movement was significantly slower in chronic LBP group with a high degree of kinesiophobia than in the group with a low degree of kinesiophobia. ${ }^{36}$ These findings may facilitate an understanding of the mechanisms whereby kinesiophobia could lead to decreased work productivity.

In the present study, we found positive correlations with depressive symptoms and presenteeism. Many epidemiological studies have indicated that depression is a predictor of the chronicity of $\mathrm{LBP}^{37} \mathrm{~A}$ large-scale study with data from the Japan National Health and Wellness Survey has indicated that chronic LBP patients with depression had a lower quality of life and work productivity. ${ }^{13}$ Moreover, a previous study on the economic impact of the health condition on work productivity has demonstrated that depression, as well as LBP, is one of the leading causes of presenteeism. ${ }^{3}$ Given that depressive symptoms were independently associated with presenteeism even after adjustment for LBP intensity in this study, the evaluation of and approach to depression might need to be reconsidered for the development of more effective strategies to improve work performance among individuals with LBP.

Sleep disturbances are common in individuals with LBP. $^{38}$ We found that shorter sleep duration was significantly associated with greater presenteeism among nursing staff with LBP. These findings were supported by previous studies, which indicated that reduced productivity was the commonly reported work performance impairment in workers with insomnia. ${ }^{39,40}$ Disturbed (inadequate) sleep has been indicated to cause daytime sleepiness, which could result in decreased productivity and accidents on the job. ${ }^{39}$ In our study, the relationship between sleep duration and presenteeism remained significant after controlling for LBP intensity and psychological distress, that were considered to be common confounders. ${ }^{11,13}$ These results imply that sleep condition is one of the key factors to improve work productivity among nursing staff with LBP, regardless of the degree of LBP intensity or psychological problems.

Among the participants in this study, only 3.3\% $(n=22)$ had been absent from work in the past year because of LBP. The low rate of absenteeism in our study was similar to that in a previous multicenter international study. ${ }^{8}$ This study revealed that the proportion of absenteeism attributed to musculoskeletal disorders was much lower in Japan than in the UK. The prevalence of absenteeism owing to LBP may depend on a different set of values or cultures between 
countries, or the type of occupation. In the present study, we did not perform statistical analysis with absenteeism as an outcome variable because the number of identified individuals with absenteeism was considerably low.

Our study found that psychological (kinesiophobia and depression) and sleep problems were associated with presenteeism. These factors could be a barrier to treatment efficacy or functional recovery. Our results encourage clinicians and occupational health staff to identify these problems prior to any intervention. The assessment of these factors at an early stage would need planning for biopsychosocial approaches focused on the modification of psychological and lifestyle factors, to improve work productivity among workers with LBP.

Our findings should be interpreted with regard to several limitations. First, approximately one-fifth of the participants had missing data in the present study. Analyses conducted after excluding individuals with missing variables could produce biased estimates and reduce precision and power. Therefore, we undertook a sensitivity analysis with multiple imputation to address the potential bias with regard to the missing data, and to determine the robustness of our findings. The results using imputed data were similar to those from the complete-case analysis. Second, we evaluated presenteeism through a self-reported questionnaire. Although absenteeism was easily evaluated by objective measures with regard to sick leave, most presenteeism instruments rely on self-reported data. ${ }^{41}$ A previous study indicated that self-reported presenteeism was strongly associated with objective measures of productivity. ${ }^{42}$ Therefore, we adopted the WPAI-GH scale in the present study; the scale is a validated, frequently used tool for the assessment of presenteeism worldwide. Third, our study was conducted at a single medical center, which could limit the generalizability of our findings. Finally, the causal relationship between presenteeism and associated factors could not be elucidated because this survey design was cross-sectional.

\section{Conclusions}

In summary, the intensity of LBP, kinesiophobia, depressive symptoms, and sleep duration were independently associated with presenteeism among nursing staff with LBP. The findings suggest that these abovementioned factors need to be considered in the development of strategies to increase work productivity among employees with LBP, as well as in the prevention of chronic LBP.

\section{Abbreviations}

LBP, low back pain; TSK-11, Tampa Scale for Kinesiophobia11; NRS, Numerical Rating Scale; BMI, body mass index; BJSQ, Brief Job Stress Questionnaire; WPAI-GH, Work Productivity and Activity Impairment-General Health.

\section{Data Sharing Statement}

The dataset analyzed during the current study is available from the corresponding author on reasonable request.

\section{Ethics Approval and Consent to Participate}

The authors assert that all study procedures were undertaken in compliance with the ethical standards of the relevant national and institutional committees on human experimentation as well as with the tenets of the Declaration of Helsinki (1964) and its later amendments until 2013. All participants provided written informed consent for study participation. This study protocol was approved by the Research Ethics Committee of Kameda Medical Center (approval no. 16-159).

\section{Acknowledgments}

We would like to thank Mrs Noriko Maruyama, Mrs Tsuyako Takanashi, Mrs Kyoko Yamamoto, and all participants at the nursing department in Kameda Medical Center for supporting the study.

\section{Funding}

This study was supported by a grant from Industrial Disease Clinical Research Grants (No. 14020301). The funder had no role in study design, data collection and analysis, decision to publish, or preparation of the manuscript.

\section{Disclosure}

The authors declare the following potential conflicts of interest: KM is a shareholder/adviser of Trunk Solution Co., Ltd. and received the following support: a research grant from the Ministry of Health, Labour and Welfare for the submitted work; grant support from Sumitomo Dainippon Pharma Co., Ltd. and Okamura Corporation; grant support, including lecture fees from AYUMI Pharmaceutical Corporation, Nippon Zoki Pharmaceutical Co., Ltd., Ono Pharmaceutical Co., LTD., Eli Lilly Japan K.K., Astellas Pharma Inc., TOTO LTD., and Eisai Co., Ltd.; lecture fees from Pfizer Japan Inc., Hisamitsu Pharmaceutical Co., Inc., Janssen Pharmaceutical K.K., Kaken Pharmaceutical Co., LTD., and Teijin Pharma Limited; and lecture fees and advisory fees 
from Shionogi \& Co., Ltd., MTG Co., Ltd., Sompo Holdings, Inc., NUVASIVE Japan, Murata Manufacturing Co., Ltd., grants and personal fees from The Association for Preventive Medicine of Japan., Shionogi \& Co., Ltd., Nippon Zoki Pharmaceutical Co., Ltd, Ono Pharmaceutical Co. Ltd., AYUMI Pharmaceutical Corporation, grants from DeNA, Murata Manufacturing Co., Ltd., Chugai Pharmaceutical Co., Ltd., Sompo Holdings, Inc, MS\&AD InterRisk Research \& Consulting, Inc, NUVASIVE Japan, Medical Data Scientist and Medical AI Device Development Organization, personal fees from Eli Lilly Japan K.K, Pfizer Japan Inc, Hisamitsu Pharmaceutical Co., Inc., and Inotech Corporation, a shareholder in and adviser for Trunk Solution Co., Ltd., outside the submitted work. HO received grants from Teijin Pharma Limited, Pfizer Inc., Eli Lilly Japan K. K., and Fujifilm Medical Co., Ltd.; grants and personal fees from AYUMI Pharmaceutical Corporation, Nippon Zoki Pharmaceutical Co., Ltd., Ono Pharmaceutical Co., LTD. MTG Co., Ltd., Sompo Holdings, Inc., and NUVASIVE Japan; and personal fees from KAKEN PHARMACEUTICAL CO., LTD, Promotion of Practical Use of AI Medical Diagnosis Support Equipment, MS\&AD InterRisk Research \& Consulting, Inc, Inotech Corporation, Chugai Pharmaceutical CO., LTD, The Association for Preventive Medicine of Japan, and Shionogi \& Co., Ltd., outside the submitted work. KM and $\mathrm{HO}$ authors report no other potential conflicts of interest for this work. The other authors have no competing interests to report.

\section{References}

1. Hartvigsen J, Hancock MJ, Kongsted A, et al. What low back pain is and why we need to pay attention. Lancet. 2018;391(10137):2356-2367. doi:10.1016/S0140-6736(18)30480-X

2. Hoy D, Brooks P, Blyth F, Buchbinder R. The epidemiology of low back pain. Best Pract Res Clin Rheumatol. 2010;24(6):769-781. doi:10.1016/j.berh.2010.10.002

3. Loeppke R, Taitel M, Richling D, et al. Health and productivity as a business strategy. J Occup Environ Med. 2007;49(7):712-721. doi:10.1097/JOM.0b013e318133a4be

4. Goetzel RZ, Long SR, Ozminkowski RJ, Hawkins K, Wang S, Lynch W. Health, absence, disability, and presenteeism cost estimates of certain physical and mental health conditions affecting U.S. employers. J Occup Environ Med. 2004;46(4):398-412. doi:10.10 97/01.jom.0000121151.40413.bd

5. Nagata T, Mori K, Ohtani M, et al. Total health-related costs due to absenteeism, presenteeism, and medical and pharmaceutical expenses in Japanese employers. J Occup Environ Med. 2018;60(5):e273-e280. doi:10.1097/JOM.0000000000001291

6. Bergstrom G, Bodin L, Hagberg J, Aronsson G, Josephson M. Sickness presenteeism today, sickness absenteeism tomorrow? A prospective study on sickness presenteeism and future sickness absenteeism. J Occup Environ Med. 2009;51(6):629-638. doi:10. 1097/JOM.0b013e3181a8281b
7. Leighton DJ, Reilly T. Epidemiological aspects of back pain: the incidence and prevalence of back pain in nurses compared to the general population. Occup Med (Lond). 1995;45(5):263-267. doi:10. 1093/occmed/45.5.263

8. Matsudaira K, Palmer KT, Reading I, Hirai M, Yoshimura N, Coggon D. Prevalence and correlates of regional pain and associated disability in Japanese workers. Occup Environ Med. 2011;68 (3):191-196. doi:10.1136/oem.2009.053645

9. Urquhart DM, Kelsall HL, Hoe VC, Cicuttini FM, Forbes AB, Sim MR. Are psychosocial factors associated with low back pain and work absence for low back pain in an occupational cohort? Clin $J \quad P a i n . \quad 2013 ; 29(12): 1015-1020$. doi:10.1097/AJP.0b013e $31827 \mathrm{ff0c0}$

10. Bernal D, Campos-Serna J, Tobias A, Vargas-Prada S, Benavides FG, Serra C. Work-related psychosocial risk factors and musculoskeletal disorders in hospital nurses and nursing aides: a systematic review and meta-analysis. Int J Nurs Stud. 2015;52(2):635-648. doi:10. 1016/j.ijnurstu.2014.11.003

11. Sadosky AB, DiBonaventura M, Cappelleri JC, Ebata N, Fujii K. The association between lower back pain and health status, work productivity, and health care resource use in Japan. J Pain Res. 2015;8:119-130.

12. Mannion AF, Horisberger B, Eisenring C, Tamcan O, Elfering A, Muller U. The association between beliefs about low back pain and work presenteeism. J Occup Environ Med. 2009;51(11):1256-1266. doi:10.1097/JOM.0b013e3181beac69

13. Tsuji T, Matsudaira K, Sato H, Vietri J. The impact of depression among chronic low back pain patients in Japan. BMC Musculoskelet Disord. 2016;17(1):447. doi:10.1186/s12891-016-1304-4

14. Tsuboi Y, Murata S, Naruse F, Ono R. Association between pain-related fear and presenteeism among eldercare workers with low back pain. Eur J Pain. 2019;23(3):495-502.

15. Luque-Suarez A, Martinez-Calderon J, Falla D. Role of kinesiophobia on pain, disability and quality of life in people suffering from chronic musculoskeletal pain: a systematic review. Br J Sports Med. 2019;53(9):554-559. doi:10.1136/bjsports-2017-098673

16. Yoshimoto $\mathrm{T}$, Oka $\mathrm{H}$, Ishikawa $\mathrm{S}$, Kokaze A, Muranaga $\mathrm{S}$, Matsudaira K. Factors associated with disabling low back pain among nursing personnel at a medical centre in Japan: a comparative cross-sectional survey. BMJ Open. 2019;9(9): e032297. doi:10.1136/bmjopen-2019-032297

17. Dionne CE, Dunn KM, Croft PR, et al. A consensus approach toward the standardization of back pain definitions for use in prevalence studies. Spine (Phila Pa 1976). 2008;33(1):95-103. doi:10.1097/ BRS.0b013e31815e $7 \mathrm{f} 94$

18. Kuorinka I, Jonsson B, Kilbom A, et al. Standardised Nordic questionnaires for the analysis of musculoskeletal symptoms. Appl Ergon. 1987;18(3):233-237. doi:10.1016/0003-6870(87) 90010-X

19. Kikuchi N, Matsudaira K, Sawada T, Oka H. Psychometric properties of the Japanese version of the Tampa Scale for Kinesiophobia (TSK-J) in patients with whiplash neck injury pain and/or low back pain. J Orthop Sci. 2015;20(6):985-992. doi:10.1007/s00776-0150751-3

20. Matsudaira $\mathrm{K}$, Inuzuka $\mathrm{K}$, Kikuchi $\mathrm{N}$, et al. Development of a Japanese version of the Tampa Scale for Kinesiophobia (TSK-J): translation and linguistic validation. Rinsho Seikei Geka (Clin Orthop Surg). 2013;48(1):13-19.

21. Kessler RC, Andrews G, Colpe LJ, et al. Short screening scales to monitor population prevalences and trends in non-specific psychological distress. Psychol Med. 2002;32(6):959-976. doi:10.1017/ S0033291702006074

22. Furukawa TA, Kawakami N, Saitoh M, et al. The performance of the Japanese version of the $\mathrm{K} 6$ and $\mathrm{K} 10$ in the world mental health survey Japan. Int J Methods Psychiatr Res. 2008;17(3):152-158. doi:10.1002/mpr.257 
23. Schaufeli W, Shimazu A, Taris T. Being driven to work excessively hard: the evaluation of a two-factor measure of workaholism in the Netherlands and Japan. Cross Cult Res. 2009;43(4):320-348. doi:10.1177/1069397109337239

24. Nakata A, Ikeda T, Takahashi M, et al. Sleep-related risk of occupational injuries in Japanese small and medium-scale enterprises. Ind Health. 2005;43(1):89-97. doi:10.2486/indhealth.43.89

25. Shimomitsu T, Haratani T, Nakamura K, et al. The Final Development of the Brief Job Stress Questionnaire Mainly Used for Assessment of the Individuals. Tokyo: Tokyo Medical College; 2000.

26. Haratani T, Kawakami N, Araki S. Reliability and validity of the Japanese version of NIOSH generic job questionnaire. Sangyo Igaku (Jpn J Ind Health). 1993;35(suppl):S214.

27. Kawakami N, Kobayashi F, Araki S, Haratani T, Furui H. Assessment of job stress dimensions based on the job demands-control model of employees of telecommunication and electric power companies in Japan: reliability and validity of the Japanese version of the job content questionnaire. Int $J$ Behav Med. 1995;2(4):358-375. doi:10.1207/s15327558ijbm02045

28. Ono Y, Yoshimura K, Yamauchi K, Momose T, Mizushima H, Asai M. Psychological well-being and ill-being: WHO subjective well-being inventory (SUBI). Jpn J Stress Sci. 1996;10:273-278.

29. Shimomitsu T. Manual for the evaluation of stress using brief job stress questionnaire. Available from: http://www.tmu-ph.ac/topics/ pdf/manual2.pdf. Accessed June 6, 2020.

30. Shimomitsu T, Odagiri Y. The brief job stress questionnaire. Occup Mental Health. 2004;12(1):25-36.

31. Reilly MC, Zbrozek AS, Dukes EM. The validity and reproducibility of a work productivity and activity impairment instrument. Pharmacoeconomics. 1993;4(5):353-365. doi:10.2165/00019053199304050-00006

32. Sterne JA, White IR, Carlin JB, et al. Multiple imputation for missing data in epidemiological and clinical research: potential and pitfalls. BMJ. 2009;338:b2393. doi:10.1136/bmj.b2393
33. White IR, Royston P, Wood AM. Multiple imputation using chained equations: issues and guidance for practice. Stat Med. 2011;30 (4):377-399. doi:10.1002/sim.4067

34. Rubin DB. Multiple Imputation for Nonresponse in Surveys. New York: Wiley; 1987.

35. Wieser S, Horisberger B, Schmidhauser S, et al. Cost of low back pain in Switzerland in 2005. Eur J Health Econ. 2011;12(5):455-467. doi:10.1007/s10198-010-0258-y

36. Osumi M, Sumitani M, Otake Y, et al. Kinesiophobia modulates lumbar movements in people with chronic low back pain: a kinematic analysis of lumbar bending and returning movement. Eur Spine J. 2019;28 (7):1572-1578. doi:10.1007/s00586-019-06010-4

37. Pincus T, Burton AK, Vogel S, Field AP. A systematic review of psychological factors as predictors of chronicity/disability in prospective cohorts of low back pain. Spine (Phila Pa 1976). 2002;27(5): E109-120. doi:10.1097/00007632-200203010-00017

38. Alsaadi SM, McAuley JH, Hush JM, Maher CG. Prevalence of sleep disturbance in patients with low back pain. Eur Spine J. 2011;20 (5):737-743. doi:10.1007/s00586-010-1661-x

39. Daley M, Morin CM, LeBlanc M, Gregoire JP, Savard J, Baillargeon L. Insomnia and its relationship to health-care utilization, work absenteeism, productivity and accidents. Sleep Med. 2009;10 (4):427-438. doi:10.1016/j.sleep.2008.04.005

40. Swanson LM, Arnedt JT, Rosekind MR, Belenky G, Balkin TJ, Drake C. Sleep disorders and work performance: findings from the 2008 National Sleep Foundation Sleep in America poll. J Sleep Res. 2011;20(3):487-494. doi:10.1111/j.1365-2869.2010.00890.x

41. Brooks A, Hagen SE, Sathyanarayanan S, Schultz AB, Edington DW. Presenteeism: critical issues. J Occup Environ Med. 2010;52 (11):1055-1067. doi:10.1097/JOM.0b013e3181f475cc

42. Meerding WJ, IJzelenberg W, Koopmanschap MA, Severens JL, Burdorf A. Health problems lead to considerable productivity loss at work among workers with high physical load jobs. J Clin Epidemiol. 2005;58(5):517-523. doi:10.1016/j.jclinepi.2004.06.016
Journal of Pain Research

\section{Publish your work in this journal}

The Journal of Pain Research is an international, peer reviewed, open access, online journal that welcomes laboratory and clinical findings in the fields of pain research and the prevention and management of pain. Original research, reviews, symposium reports, hypothesis formation and commentaries are all considered for publication. The manuscript management system is completely online and includes a very quick and fair peer-review system, which is all easy to use. Visit http:// www.dovepress.com/testimonials.php to read real quotes from published authors. 